\title{
USE OF THERAPEUTIC PLASMAPHERESIS IN ETHYLENE DIBROMIDE INTOXICATION
}

Dolly Joseph, S. Abbas, P. Joshi, P. Bajpai, S. Maheshwari, S. Chelawat, S. Dudve, R .K. Jha

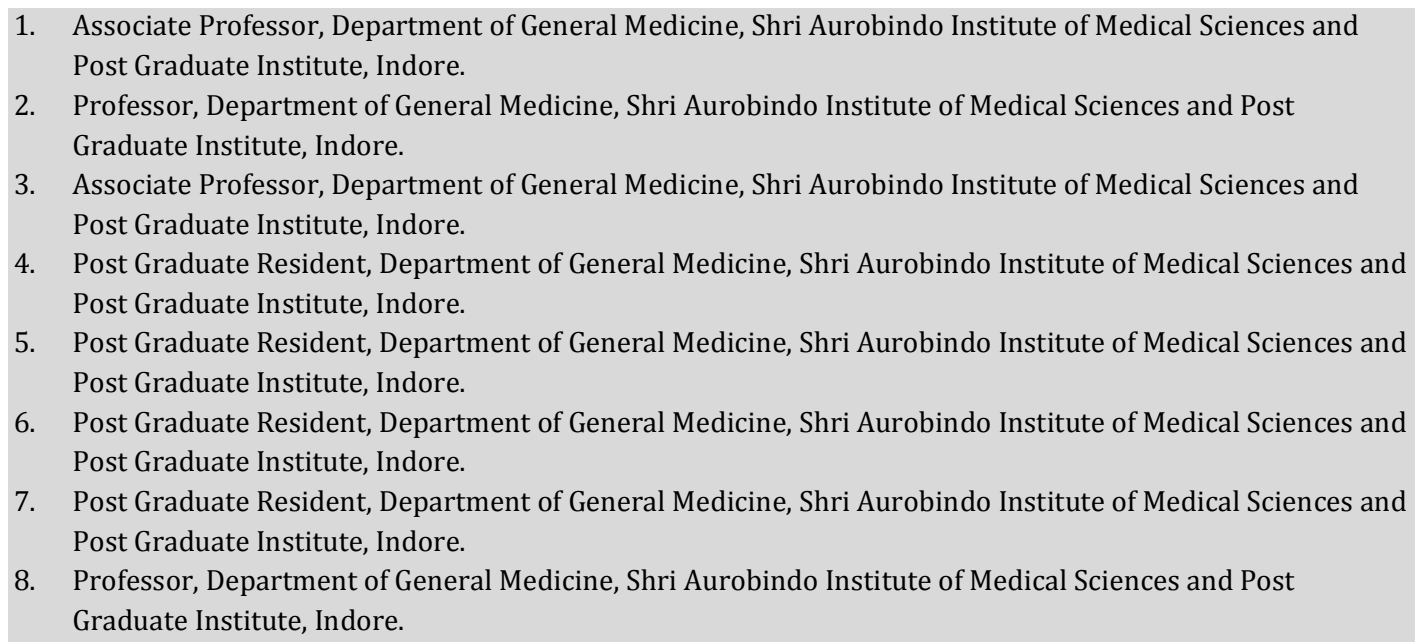

\section{CORRESPONDING AUTHOR}

Dr. Dolly Joseph,

Shri Aurobindo Institute of Medical Sciences and post graduate institute,

Sanwer Road, Bhanwrasala Tehsil Sanwer,

District Indore (M.P.) India 453111,

E-mail: shaji_dolly22@yahoo.co.in

Ph: 00919826755137

ABSTRACT: In view of high incidence of mortality with Ethylene dibromide poisoning, a retrospective study was conducted, with objectives to compare demographic, toxicological \& clinical outcome in patients treated conservatively and those who underwent therapeutic plasmapheresis.

Records of Forty patients from central India region, with ingestion of ethylene dibromide admitted over last six years were reviewed.The majority of patients were less than 30 years (77.5\%). Gastrointestinal symptoms (87.5\%) were predominant at presentation. Amongst biochemical parameters hepatotoxicity was seen in 26 (65\%), cardio toxicity in 19 (47.5\%) and renotoxicity was seen in 4 (10\%). Fourteen patients (35\%) had normal biochemical parameters. Out of 26 patients with hepatotoxicity, 13 were treated conservatively whereas 13 underwent plasmapheresis. In conservative group the mean liver enzymes level were 937.6 I.U./ml (range 96-3717) ,while in plasmapheresis group it was 2587.6 I.U./ml (126$11700)$. The mortality rate was $5 / 13(38.4 \%)$ in conservatively treated patients as compared to $3 / 13(23 \%)$ in plasmapheresis group. Mean length of hospital stay was 5.4 days (range 2-20 days).Best outcome was when the patient presented to the hospital within six hours of ingestion. Death occurred anywhere between twenty hours and five days.

Ethylene dibromide is highly toxic substance with a reported mortality rates as high as $30-60 \%$.In our study we found a $15.4 \%$ decrease in mortality in plasmapheresis group as compared to conservative. Thus plasmapheresis may be considered an important treatment modality for ethylene di bromide poisoning. 
KEY WORDS: Hepatotoxi,city, mortality, poison

INTRODUCTION: Acute poisoning with agriculture pesticide is worldwide health problem. Ethylene Di-bromide (EDB) is widely available in Indian market as $5 \mathrm{ml}$ ampoule \& has been used as a bulk grain fumigant to protect against insect, pests and nematodes. It is a colorless liquid at ambient temperature, which decomposes in the presence of heat and light to ethylene and bromine ions. $(1,2)$

On ingestion its systemic effects are seen immediately as abdominal pain, nausea, vomiting \& diarrhea. It causes necrosis of he pacyte \& tubular epithelium of proximal convoluted tubule in kidney resulting in metabolic acidosis. (1)The mortality in first $48 \mathrm{hr}$ is due to circulatory failure while later on due to hepatic \&/or renal failure (2)

As there is no antidote available and because of latent period in the development of symptoms ensuing hepatic or renal failure, use of therapeutic detoxification by plasmapheresis may be considered. Mortality rate as high as 30\% to $60 \%$ are reported in various Indian studies. ${ }^{(3-4)}$ we tried to find out the clinical outcome in Plasmapheresis treated group, as there is no study in Indian literature suggesting its uses and effectiveness.

MATERIAL AND METHODS: A total of forty cases of Ethylene Di-bromide poisoning, admitted over Jan 2006 -Dec 2012 in our institution, were included in this retrospective descriptive study after obtaining approval from institutional research committee. Patients who were admitted with a history of ingestion of ethylene dibromide, but discharged against medical advice were excluded from this study.

All patients received gastric decontamination followed by charcoal, to further inhibit the absorption of poison from gut. Their biochemical parameters were recorded sequentially for initial 2-3 days. Symptomatic and supportive care in the form, antibiotics, dialysis for those with renal shutdown and transfusion of fresh frozen plasma were given to those with bleeding manifestations. Patients with deranged liver function test (liver enzymes> 2 times or PT with INR >1.5 times) or renal functions were subjected to plasmapheresis after taking written consents. During the procedure of therapeutic plasmapheresis 2-2.5 liters of plasma were removed in each setting of plasmapheresis using a Fresinius medical care 4008s machine by centrifugation technology via an intravenous subclavian or femoral vein catheter access and is replaced by either intravenous fluids like ringer lactate, human albumin \&/ or fresh frozen plasma.

DATA ANALYSIS: Data of all patients were reviewed for the following-

1. Demographic profile.

2. Clinical profile.

3 Toxicological profile.

4. Outcome in conservative and plasmapheresis group.

OBSERVATIONS: Majority of patients were less than 30 years $31 / 40$ (77.5\%) with female predominance. The median age was 25 years (range17-50) (Table 1 ) 
Fourteen patients did not show any features of toxicity or deranged biochemical parameter. Remaining 26 patients (65\%) developed various kinds of organ toxicities (Table 4.) Acute toxic hepatitis with various degree of severity were observed in $26 / 40$ patients (65\%) (Table-5).These was in the form of jaundice, elevated liver enzymes, increased prothrombin time and bleeding time. Out of 26 patients who had deranged biochemical parameters 13 patients were treated conservatively and remaining 13 patients were subjected to therapeutic plasmapheresis.

Though both groups differs as shown by the mean liver enzymes level, which were 937.6 I.U./ml(range 96-3717) in conservative group, and 2587.6 I.U./ml (126-11700) in plasmapheresis group. Nephrotoxicity and cardiotoxicity were seen in 4/40 patients (10\%),19/40(47.5\%) patients respectively.(Table 4) We observed An overall mortality of $10 \%, 13 \%, 50 \%$ and $100 \%$ with ingestion of $1,2,3$, and $>4$ ampoule $(1 \backslash$ ampoule $=5 \mathrm{ml}$ ) (Table 3) Overall mortality was $5 / 13(38.46 \%)$ in conservative group where as $3 / 13(23 \%)$ in plasmapheresis group Time from ingestion to death was anywhere between less than twenty hours to four days in our study.

All the patients underwent at least two session of plasmapheresis, and one patient was subjected to haemodialysis. Adverse reaction related to plasmapheresis was observed in two patients who received FFP during the procedure But those reactions did not results in cancellation of procedure. The average number of plasmapheresis session was1.84 per patient.

DISCUSSION: Ethylene Di-bromide readily penetrates skin clothing and other protective material. Its route of absorption can be either by inhalation, ingestion and de rmal ${ }^{(1)}$. Our study showed suicidal intent among the younger age group <30years (77.5\%) with majority being female $17 / 31(54.83 \%)$. The systemic features of toxicity were mainly gastrointestinal like nausea, vomiting (86.4\%), and abdominal pain (81\%) to begin with, followed by progressive dysfunction of other organ system like liver and kidney as was seen in other Indian studies ${ }^{(3-5)}$.

Ingestion of small amount of EDB could be nonfatal,(6) but exposure to more than $5 \mathrm{ml}$ is

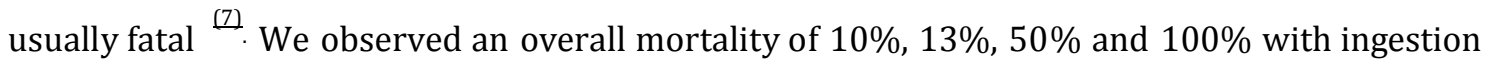
of $1,2,3$, and $>4$ ampoule respectively ( 1 ampoule $=5 \mathrm{ml}$ ) (Table 3 )

Plasmapheresis or therapeutic plasma exchange is an extra corporeal technique designed for removal of large molecular weight substances highly bound to plasma protein. Besides treating for removal of autoantibody (primary biliary cirrhosis, acute humoral rejection post hepatic transplant) its role is rapidly increasing in treatment of poisoning with drugs ( like Phenytoin, Theophylline, Tri- and Tetra-cyclic antidepressant, L-thyroxin, Verapamil, Diltiazem, Carbamazepine etc,) ,heavy metal intoxication(Mercury and Vandate), snake bite and Phalloid mushroom intoxication, for which there is no antidote(8-15) According to the volume of distribution, protein binding and solubility in water, different methods are chosen. Haemodialysis is best for water soluble and dialyzable substances (ethylene glycol, methanol), Haemoperfusion (Charcoal) is used for Phenobarbital. Plasmapheresis is the best option for substance that is not removed effectively by either haemodialysis or haemoperfusion.(8) Documentation of removal of toxicsubstances from blood remains the major objective for the effectiveness of Plasmapheresis in any intoxication. EDB is covalently bound to albumin(16) and in plasmapheresis; toxic metabolite 
2 bromoacetaldehyde bound to plasma protein is removed, before it causes organ damage like nephrotoxicity and hepatotoxicity. In EDB, serum bromide level can be used to document that exposure did occur but bromide levels do not accurately predict the clinical course $^{(3)}$

Plasmapheresis has been observed in reducing the mortality from as high as 35$50 \%$ with conventional therapy to $20 \%-10 \%$ in phalloid mushroom intoxication and the best therapeutic results can be expected when the detoxification technique are applied within first 36-48 hours. ${ }^{(8-10)}$

In our study we observed a reduction in mortality rate from $38.46 \%$ to $23 \%$ in EDB intoxication. Though both groups were noncomparable, as severe hepatotoxicity (liver enzymes $>25$ times) were observed in $38.4 \%(5 / 13)$ of patients in plasmapheresis group with mortality rate of $60 \%$, while in conservative group it was $23 \%(3 / 13)$ with $100 \%$ mortality rate. Thus there is a reduction in mortality rate of $40 \%$ in plasmapheresis group with severe hepatotoxicity. The mean liver enzyme level were 937.6 I.U. / ml (range 963717) in conservatively treated patients, while in plasmapheresis group the mean liver enzyme were 2587.6 I.U./ml (126-11700). There is no controlled study on the usefulness of Plasmapheresis in any particular intoxication because of lack of large reported series .Case reports are published instead, and depending on the severity of the reported intoxication and on the Plasmapheresis protocol used; either dramatic improvement orno effect is reported. Documentation of removal of the toxic substance from the blood therefore remains the major objective judgment of the effectiveness of Plasmapheresis in any particular type of intoxication

CONCLUSION: Ethylene dibromide is highly toxic substance; up to one ampoule may be lethal if not treated immediately. With mortality rate as high as up to $60 \%$, therapeutic plasmapheresis can be considered as a treatment option. We observed a significant reduction in mortality in plasmapheresis group especially those with severe hepatotoxicity.

\section{REFRENCES:}

1. HSDB. Hazardous Substances Data Bank, National library of medicine 2004, MICROMEDEX. CO. USA.

2. Sharma VK, Sharma AK, Satpathy DK. Ethylene dibromide poisoning homicide or suicide; JIAFM,2004:26(4).160-161

3. Neelima Singh, Jatav OP, Gupta RK, Tailor MK, \& Jain R. Outcome of Sixty Four cases of Ethylene Di-bromide ingestion treated in Tertiary Care Hospital; J Assoc Physicians of India Dec.2007 p. 842-845

4. Raman PG, Sain T. Clinical profile of ethylene dibromide (EDB) Poisoning. J Assoc Physicians India 1999;47:712-3

5. Bardale Rajesh, Dixit P. G., Shrigiriwar Manish. Clinico-pathological profile of Ethylene dibromide poisoning. Journal of Indian Academy of Forensic Medicine Year: 2006, Volume: 28, Issue: 3

6. Singh S, Gupta A, Sharma S, Sud A, Wanchu A, Bambery P. Non-fatal ethylene dibromide ingestion. Hum Exp Toxicol 2000;19:152-3

7. Letz GA, Pond SM, Osterloh JD, Wade RL, Becker CE. Two fatalities after acuteoccupational exposure to ethylene dibromide. J.Am Med Assoc 1984 ;252:2428-3 
8. Vesselin D.Nenov, Petko Marinov, Julia Sabeva and Dimitar S. Nenov. Currentapplications of plasmapheresis in clinical toxicology. Nephrol Dial Transplant (2003) 18[Suppl 5]: v56-v58.

9. Yildirim C., Bayraktaroğlu Z., Gunay N., Bozkurt S., Köse A., Yilmaz M..The use of therapeutic plasmapheresis in the treatment of poisoned and snake bite victims: An academic emergency department's experiences. J Clin Apher. 2006 Dec;21(4):219-23.

10. Jander S, Bischoff J Treatment of Amanita phalloides poisoning: I Retrospective evaluation of plasmapheresis in 21patients. Ther Apher 2000; 4: 303-30.

11. Roger L.White ,William R Garnett, Jody H. Allen, Berry J. Kline and David E. Sharp . Phenytoin removal during plasma exchange, J Clin Apher.3:147-150(1987).

12. A Duzova,E Baskin,Y Usta and S. Ozen. Carbamazepin poisoning : Treatment with plasma exchange. Hum. Exp. Toxicol (2001)20,175-177.

13. Robert Kramer, Payson Oberg -Higgins, Louis Russo, John H. Braxton. Hepin induced thrombocytopenia with thrombosis syndrome managed with plasmapheresis. Interactive cardiovascular and thoracic surgery 8 (2009)439-441.

14. Alallam Alallam, D.Barth, EJ Heathcote. Role of plasmapheresis in treatment of severePruritus in pregnant patient with primary billiary cirhhosis: Case report. Can. J.gastroenterol 2008;22(5):505-507.

15. Nassim Kamar, Laurence Lavayssiere, Fabrice Muscari, Janick Selves et al Eay plasmapheresis and rituximab for acute humoral rejection after ABO compatible liver transplant. World J. gastroenterol 2009 July 21:15(27); 3426-3430.

16. Bhupendra S. Kaphalia, and G.A.S. Ansari.Covalent binding of Ethylene dibromide and its metabolites to albumin. Toxicology Letters Volume 62, Issues 2-3, September 1992, p. 221-230.

TABLE 1: DEMOGRAPHIC PROFILE

\begin{tabular}{|c|c|c|c|}
\hline AGE(YEARS) & MA & FEMALE & TOTAL \\
\hline 11 & 5 & 6 & 1 \\
\hline 21 & 9 & 1 & 2 \\
& 6 & 2 & 0 \\
\hline 31 & 0 & 1 & 1 \\
\hline 41 & $\mathbf{2}$ & $\mathbf{2}$ & $\mathbf{4}$ \\
\hline TOTAL & $\mathbf{n}$ & $\mathbf{n}$ & $\mathbf{n}$ \\
\hline
\end{tabular}

TABLE 2: Clinical Characteristics of Ethylene Dibromide Poisoning

\begin{tabular}{|c|c|c|}
\hline SYMPTOM & NUMBER OF PATIENTS & PERCENTAGE \\
\hline Abdominal pain & 33 & $82.5 \%$ \\
\hline Nausea \& vomiting & 35 & $87.5 \%$ \\
\hline Drowsiness & 10 & $25 \%$ \\
\hline Oliguria & 4 & $10 \%$ \\
\hline Haematmesis & 3 & $7.5 \%$ \\
\hline
\end{tabular}


TABLE: 3 Number of ampoules consumed and mortality pattern

\begin{tabular}{|c|c|c|c|c|c|}
\hline \multirow{2}{*}{ AMPOULES } & \multicolumn{2}{|c|}{ PLASMAPHERESIS } & \multicolumn{2}{c|}{ CONSERVATIVE } & \multirow{2}{*}{ TOTAL } \\
\cline { 2 - 5 } & SURVIVED & EXPIRED & SURVIVED & EXPIRED & TOT \\
\hline 1 & 4 & 1 & 12 & 1 & 19 \\
\hline 2 & 4 & 0 & 9 & 2 & 15 \\
\hline 3 & 2 & 1 & 0 & 0 & 3 \\
\hline 4 & 0 & 1 & 0 & 1 & 2 \\
\hline 5 & 0 & 0 & 0 & 1 & 1 \\
\hline & $\mathbf{1 0}$ & $\mathbf{3}$ & $\mathbf{2 2}$ & $\mathbf{5}$ & $\mathbf{4 0}$ \\
\hline
\end{tabular}

TABLE 4: TOXICITY PROFILE in Ethylene Dibromide Poisoning

\begin{tabular}{|c|c|c|c|}
\hline & CONSERVATIVE & PLASMAPHERESIS & TOTAL \\
\hline HEPATIC & 15 & 11 & 26 \\
\hline RENAL & 4 & 6 & 10 \\
\hline CARDIAC & 7 & 12 & 19 \\
\hline HEMATOLOGICAL & 1 & 2 & 2 \\
\hline C.N.S & 2 & 2 & 2 \\
\hline $\begin{array}{c}\text { NO BIOCHEMICAL } \\
\text { ABNORMALITY }\end{array}$ & 14 & 0 & 14 \\
\hline
\end{tabular}

